\title{
Technically measured compositional physical work demands and prospective register-based sickness absence (PODESA): a study protocol
}

Sofie Dencker-Larsen ${ }^{1 *}$ (D) Charlotte Lund Rasmussen ${ }^{1,2}$, Sannie Vester Thorsen ${ }^{1}$, Els Clays ${ }^{3}$, Thomas Lund ${ }^{4}$, Merete Labriola ${ }^{5}$, Ole Steen Mortensen ${ }^{2,6}$, Marie Birk Jørgensen", Nidhi Gupta',

Charlotte Diana Nørregaard Rasmussen ${ }^{1}$ and Andreas Holtermann ${ }^{1,8}$

\begin{abstract}
Background: Various physical work demands are shown to be associated with sickness absence. However, these studies have: (a) predominantly used self-reported data on physical work demands that have been shown to be inaccurate compared with technical measurements, (b) principally focused on various physical work demands in 'isolation', i.e. ignoring their co-dependency - compositional nature -, and (c) mainly used register data on long-term sickness absence. The present article describes the protocol of a study with the objective of investigating the association between technically measured compositional data on physical work demands and prospective long- and short-term register-based data on sickness absence.

Methods: The technically measured compositional Physical wOrk DEmands and prospective association with registerbased Sickness Absence study (PODESA)' comprises data from two Danish cohorts (NOMAD and DPhacto) primarily on blue-collar workers. In the PODESA cohort, data on 1108 workers were collected at baseline (between 2011 and 2014). The cohort data comprise, e.g., self-reported information on descriptives, lifestyle, workday, and health, as well as accelerometer-based measurements of physical work demands (physical activity, movements, and postures). These baseline measurements are linked with prospective register-based data on sickness absence for up to four years after baseline. The prospective association between physical work demands and sickness absence will be analysed using a Compositional Data Analysis approach.

Discussion: PODESA provides a unique possibility of unravelling which combinations of physical work demands are associated with prospective sickness absence. PODESA employs technically measured information on physical work demands (taking into account the compositionality of physical work demand data) and prospective sickness absence data. The findings from PODESA can be used to develop strengthened preventive interventions for sickness absence. Results are expected in 2019-2021.
\end{abstract}

Keywords: Accelerometers, Compositional data analysis (CoDA), Physical activity at work, Sick-leave, Time-use epidemiology

\footnotetext{
* Correspondence: sdl@nrcwe.dk

${ }^{1}$ National Research Centre for the Working Environment, Lersø Parkallé 105,

Copenhagen, Denmark

Full list of author information is available at the end of the article
}

(c) The Author(s). 2019 Open Access This article is distributed under the terms of the Creative Commons Attribution 4.0 International License (http://creativecommons.org/licenses/by/4.0/), which permits unrestricted use, distribution, and 


\section{Background}

Sickness absence is costly to workers, employers, and society. In a 2008 report, the Danish Ministry of Employment presents that the yearly cost of sickness absence corresponds at least 37 billion DKK in unproductive wages and sickness absence subsidies alone in Denmark [1, 2]. Moreover, sickness absence is a considerable risk factor for workers permanently exiting the labour market [3-5].

Physical work demands - physical activity, movements, and postures at work - are amongst the dominant causes of long-term sickness absence [6, 7]. Physical work demands such as stationary standing $[7,8]$, sitting $[9,10]$, forward bending of the trunk $[7,8,11]$, and arm elevation $[7,8,11]$ have been shown to be associated with sickness absence $[6-8,12,13]$.

However, there are three overall issues with the research literature on physical work demands and sickness absence: 1) physical work demands have mainly been measured using self-reports, 2) the analytical methods used in previous studies on physical work demands have predominantly ignored the compositional nature of the data, and 3) studies analysing sickness absence using register data, have generally omitted short-term sickness absence.

First, previous studies on physical work demands included self-reported information on physical work demands [14, 15], that have been presented to be less accurate than technical measurements [16-20] (e.g., for sitting time). Such technical measurements are in this case accelerometers attached to the body of study participants; accelerometers use accelerations of the body [21] to measure physical activity, movements, and postures. Therefore, future studies investigating the association between physical work demands and sickness absence are likely to strengthen the field of research when using accelerometer measurements.

Second, the vast majority of existing studies analysing physical work demands have investigated the effect of each physical work demand 'in isolation' of other physical work demands. For example, by, e.g., investigating the health effects of sitting time without taking into account the time spent on all remaining demands such as standing, or resting. Time-use on various physical work demands is constrained or fixed by nature - summing up to $100 \%$ - (or for example $8 \mathrm{~h}$ ). Therefore, the proportion of time spent on physical work demands carries relative information, is co-dependent. Addressing this special property of data on physical work demands requires special statistical methodology - Compositional Data Analysis (CoDA) [22-25]. Only recently, a limited amount of studies have used Compositional Data Analysis approaches to address the compositional property of physical work demands [24, 26]. However, none of them have investigated the association between physical work demands and sickness absence. Therefore, future studies investigating the association between time spent on various physical work demands and prospective sickness absence using a Compositional Data Analysis approach are needed.

Third, the previous studies have often used sickness absence using self-reports that have less validity than sickness absence information from national registers in Nordic countries [27]. Studies using national register-data on sickness absence have predominantly used long-term sickness absence (see, e.g., [8, 12, 28-30]). Nevertheless, physical work demands has also been associated with short-term sickness absence [31], and, like sickness absence overall [32-35], is likely to be placing a considerable economic burden on workplaces and society. Studies investigating both long-term and short-term sickness absence register data are thus warranted.

\section{Aim}

The purpose of the present article is to present the protocol for the "The technically measured compositional Physical wOrk DEmands and prospective association with register-based Sickness Absence study (PODESA)'. Specifically, to counter the above-presented challenges of previous studies, PODESA will be the first study to investigate the association between technically measured compositional data on physical work demands and prospective register-based data on short- and long-term sickness absence. PODESA will investigate the following hypothesis:

The composition of physical work demands is associated with prospective sickness absence.

\section{Design}

\section{Data}

PODESA will combine accelerometer-based data on physical work demands and other required data from the two Danish cohorts 'New method for Objective Measurements of physical Activity in Daily living (NOMAD)' [36] and 'the Danish Physical Activity cohort with Objective measurements (DPhacto)' [37]. These merged data will then be linked with prospective data on sickness absence using two types of extensive sickness absence registers.

\section{The PODESA cohort}

The PODESA cohort consists of the merged NOMAD and DPhacto cohorts that were collected using almost identical data gathering procedures. Both cohorts included predominantly blue-collar workers from Denmark. Data collection on the NOMAD and DPhacto cohorts was conducted from 2011 to 2012 [16] and 2012 to 2014 [37], respectively. The NOMAD and DPhacto cohorts are described in detail elsewhere [37, 38]. In short, The 
NOMAD cohort included workers from seven workplaces within fields such as cleaning, construction, transport, and the health service sector [38], and the DPhacto cohort included workers from 15 workplaces within cleaning, manufacturing and transport industries [37, 39].

As shown in Table 1, we have combined three types of data from the NOMAD and DPhacto cohort into the PODESA cohort: 1) accelerometer data, 2) questionnaire data, and 3) health check data. The data from the PODESA cohort will be merged with detailed register data on sickness absence. At the time of submitting the manuscript, the authors had not yet conducted analyses of associations between exposure variables (PODESA cohort) and outcome variables (sickness absence data) (except, making the sample flowchart). This strategy was chosen to minimise bias of hindsight in the protocol.

\section{Technical measurements of physical work demands}

PODESA employs accelerometer-based data on physical work demands - physical activity, movements, and postures at work. Eligible participants of the cohort were asked to wear up to four triaxial ActiGraph accelerometers (GT3X+, Florida, U.S.A). The accelerometers were located at the dominant arm, upper back, hip, and right thigh. Participants wore the accelerometers for up to four consecutive workdays [36, 37]. Moreover, participants were asked to fill-in a short paper-based diary reporting time at work, time of going to bed and out of bed, non-wear time, and reference time ( $15 \mathrm{~s}$ of standing still to calibrate the accelerometer); and to remove the accelerometer if they experienced discomfort or itching [37]. Non-wear-time was determined by the following premises: (a) the software showed $>60 \mathrm{~min}$ of zero counts per minute, (b) the participant reported non-wear periods, and (c) visual inspection revealed artefacts or missing data [36].

The accelerometers were initialized and the data from the accelerometers were downloaded using the Actilife Software version 5.5 [40]. In brief, the accelerometer data are low-pass filtered with a $5 \mathrm{~Hz} 4$ th order Butterworth filter. Thereafter they were split-up into $2 \mathrm{~s}$ intervals with an overlap of $50 \%[36,41]$.

Next, using a custom-made MATLAB program Acti4 [41, 42] (The National Research Centre for the Working Environment, Copenhagen, Denmark), the accelerometry data were analysed to obtain comprehensive information of physical work demands such as various physical activities (e.g., standing, walking, lying, cycling, stair climbing, and running) and postures (e.g., sitting, standing, forward bending, and arm elevation at various degrees). The Acti4 software has been shown to provide valid estimates of physical activities with high specificity and sensitivity $>99 \%$ under standardised and semi-standardized conditions [41]; in a free-living setting, Acti4 has been shown to have a specificity and sensitivity> $80 \%$ [43].
We will use the following specifications: We average daily time-use for all valid measured days on various physical work demands. Additionally, as physical work demands and physical activity behaviour at leisure are co-dependent [23], we expect to include information of time-use on various physical activity behaviours at leisure (i.e., sedentary behaviour, physical activity) and sleep domain (time in bed) summarized on all valid measured days. We consider a day to be valid if it comprises a valid work, leisure and sleep domain. A work and leisure period is considered valid if it comprises $\geq 4 \mathrm{~h} /$ day of accelerometer wear-time or $\geq 75 \%$ of the average wear-time across days (see, e.g., [24, 26, 44]). A time in bed period is considered valid if it comprises at least $4 \mathrm{~h}$ (see, e.g., [44]). To assess when participants were at work or at leisure or spent time-in-bed, we use information from the diaries.

\section{Measurements of sickness absence}

PODESA will use data on sickness absence from two registers: 1) the DREAM register (comprising mainly long-term sickness absence) and 2) the 'Danish register of work absences' (covering a smaller portion of workers than the DREAM register, but includes also short-term sickness absence). Both are obtainable from Statistics Denmark [45-47].

First, the DREAM register is an acronym in Danish for 'the Register-based Evaluation of Marginalization' [3]). This Danish Ministry of Employment register is continuously updated and documented by The Danish Agency for Labour Market and Recruitment [48, 49]. It encompasses all episodes of sickness absence from work subsidized by the state. Typically these are granted after 30 days of sickness absence; thus excluding the often shorter periods of sickness absence which are not subsided. The DREAM register is widely used in studies measuring sickness absence (see, e.g., [8, 12, 28-30]).

Second, the 'Danish Register of Work Absences' (technical name used by Statistics Denmark: 'FRAN', 'FRPE') $[45,46,50]$ includes sickness absence periods from work, including days on sickness absence [51]. This register contains data from all public employees and a considerable sample ( $N=2600$ companies) of privately owned companies, a representative sample of private companies with 10 to 249 employees, and all private companies with 250 or more employees. Private companies with 10 to 249 employees are sampled yearly (therefore, some workers at, e.g., midsize private companies are excluded from this register). Thus, this register includes shorter sickness absence spells for a limited amount of workers. Research studies using the 'Danish Register of Work Absences' are scarce (for such studies, please see [52, 53]).

From the above-mentioned registers, PODESA has access to prospective data on sickness absence since the 
Table 1 Data-merging strategy in PODESA

1) Constructing the PODESA cohort

Data from the NOMAD and DPhacto cohorts have been combined into the PODESA cohort containing three types of data:

Accelerometer data

Identical accelerometer hardware and software was used to measure physical work demands in both the NOMAD and in the DPhacto cohorts, making the two cohorts highly comparable; thus, the accelerometer data were added in a simple merge.

Questionnaire data

Health check data

The majority of survey items from the questionnaires in the NOMAD and DPhacto cohorts are identical or comparable (77 items) enabling adding them in a simple merge. However, for the minority of survey items which were not identical, and merely similar, we assessed the comparability of the items and possible modification. Specifically, four items had accordance of question wording but dissimilar response scale size (e.g. nine versus ten categories), and five items that differed in wording (e.g. used non-identical time frames). A total of 14 items were non-comparable and therefore not merged.

For similar, but not identical, survey items, the following procedure was used: firstly, based on findings from the literature within the field, we evaluated whether dissimilarities in wording or response scales could influence the answers, and secondly, using descriptive statistics we assessed the answer distribution in both cohorts. Similar items were merged if the literature indicated no difference in answers due to wording or response scale of the items and if the answer distribution on the items was similar in the two cohorts.

The questionnaire data contain, e.g., background information to be used as covariates (such as age, sex, smoking status, alcohol intake).

(An overview of the merging of the questionnaire data is available upon request)

The health check data from the two studies derive from a health check and a physical testing session at baseline conducted by trained research professionals. Because identical health check procedures were followed in the NOMAD and DPhacto cohorts that are the basis of the PODESA cohort, we added the health check from each study in a simple merge.

The health check data includes data on, e.g., height, weight, hip and waist circumference, percentage of body fat, blood pressure, maximal oxygen uptake, maximal hand grip strength, back extension endurance and back flexibility.

2) Combining the PODESA cohort with register data on sickness absence

Data from the PODESA cohort will be linked with two types of register data on sickness absence

We combine the PODESA cohort with register data from two registers:

Register data on long-term In addition to the PODESA cohort data, we sickness absence add register data on primarily long-tern
Table 1 Data-merging strategy in PODESA (Continued)

sickness absence from the DREAM register dataset which includes weeks of subsidized sickness absence spells (typically granted after 30 days of sickness absence).

Register data on shortterm sickness absence

We also add register data including shortterm sickness absence from the 'Danish Register of Work Absences' which includes daily employer-reported sickness absence.

worker baseline measurements (which vary between 2011 and 2014) with up until four years of follow-up sickness absence data (2011-2015).

\section{Study population}

Figure 1 displays the number of participants in PODESA $(N=1108)$. A total of 391 and 2107 workers in the NOMAD cohort and the DPhacto cohort, respectively, were invited to participate. Of the 2498 invited participants, 1422 workers either handed in the questionnaire, underwent a health check at baseline, or both. Of these, a total of 1108 study participants fulfil the inclusion criteria and will be included in the PODESA cohort. Reasons for exclusion were: being sick, being on holiday, being pregnant, and 'unknown' $(N=6)$; holding a management position $(N=17)$, being a student $(N=14)$, and not wearing the accelerometers on a workday $(N=47)$.

Furthermore, the PODESA cohort will be linked with data on sickness absence for up to four years of follow-up. In the flowchart in Fig. 1, we base the flowchart $N$ on workers with at least one year follow-up data from the sickness absence registers. Of the $N=1108$ included workers in the PODESA cohort, there is sickness absence data on $N=1079$ workers from the DREAM register, and $N=726$ workers from 'The Danish Register of Work Absences'.

\section{Statistical analyses}

We expect to analyse the association between time-use in various physical work demands and prospective long-term sickness absence primarily using regression models such as time-to-event analyses based on a Compositional Data Analysis approach. This entails several steps: First, we will transform the 24-h compositional data on physical work demands and physical activity behaviour at leisure using an appropriate log-ratio method. Second, depending on the analytical definition of the outcome, we will adopt time-to-event methods (as the main analyses, we expect to use Cox time-to-event regression on sickness absence data from the DREAM sickness absence register). The models will be adjusted for variables such as age, sex, body mass index, smoking status (for similar covariates, please see, e.g., [8]). Third, to enable understanding how time spent in various postures and movements at work is associated with 


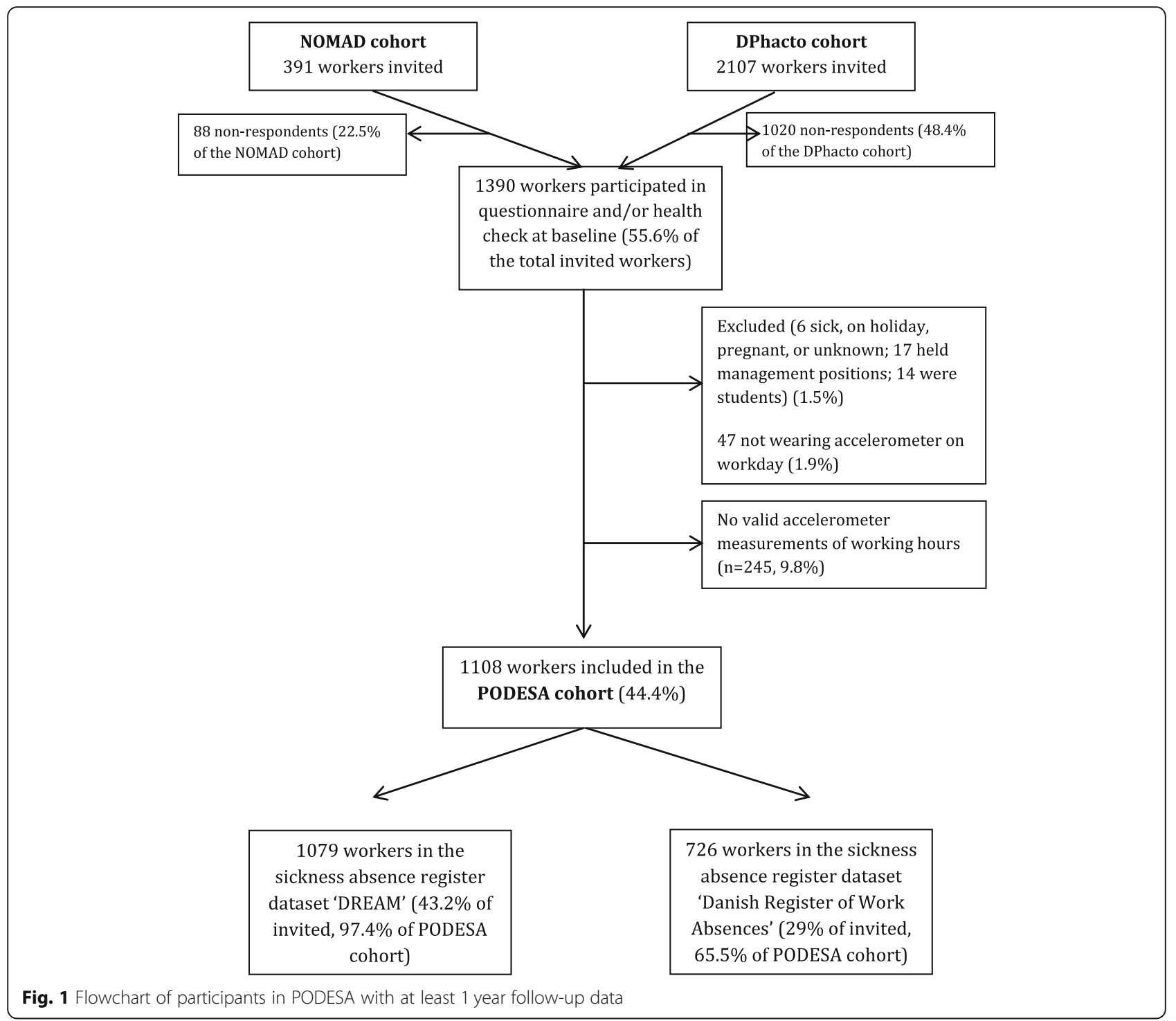

prospective sickness absence, we expect to use isotemporal substitution models [54] indicating association between reallocation of time-use in various physical work demands and the change in probability of prospective sickness absence.

Moreover, having access to detailed longitudinal register data from the two types of sickness absence registers enables us to code the outcome variable in several ways, such as binary, percentage of sickness absence, trajectories, and time-to-event, thus also enabling additional analyses further unravelling the relationship between physical work demands and sickness absence.

\section{Discussion}

The PODESA study comprises high quality data, including technical measurements of physical work demands from accelerometer data, which provide a more precise depiction compared with, e.g., self-reports. Furthermore, the combined PODESA cohort data and sickness absence register data enable us to conduct analyses not only on the links between singular exposures and sickness absence, but also on the association between relative time-use on specific physical work demands and prospective sickness absence, e.g., using Compositional Data Analysis. Additionally, having access to prospective sickness absence data on both short-term and long-term sickness absence enables us to exploit the longitudinal nature of the data in analyses using, for example, trajectories or time-to-event analyses.

Conversely, there are also potential weaknesses to discuss. First, despite having high-quality data on physical work demands, the PODESA cohort has no objectively measured data on changes in physical work demands over time; if there is a non-random change, 
e.g., due to an organizational change or change in worker instructions at the workplace, it could affect the prevalence and timing of prospective sickness absence. Second, as the cohort data stem from a Danish context, generalizability of findings to other countries in terms of, e.g., inter-country variations in occupational policy is limited. Third, as the majority of participants in the study are blue-collar workers, the findings will primarily be generalizable to this group of workers.

The findings from PODESA can be used to develop improved preventive workplace interventions for sickness absence. For instance, if the results show which combinations of physical work demands may increase - and which may decrease - the risk of sickness absence, this information can be used to design better future preventive workplace interventions for sickness absence. Results from PODESA are expected in 2019-2021.

\section{Abbreviations}

DPhacto: The Danish Physical Activity cohort with Objective measurements; DREAM: A Danish acronym for 'the Register-based Evaluation of Marginalization; NOMAD: New method for Objective Measurements of physical Activity in Daily living; PODESA: The technically measured compositional Physical wOrk DEmands and prospective association with register-based Sickness Absence study

\section{Acknowledgements}

The authors wish to thank Jan Høgelund for sharing his insights into sickness absence data registers.

\section{Funding}

The PODESA study is funded by the Danish Working Environment Research Fund (01-2015-09). The funders had no role in study design, data collection and analysis, decision to publish, or preparation of the manuscript.

\section{Availability of data and materials}

Due to Danish data protection legislation concerning research data, and register data in particular, the dataset cannot be made openly available. However, a large part of the data (the NOMAD cohort data and the DPhacto cohort data) is available to researchers in anonymised form through the Danish Data Archive.

\section{Authors' contributions}

SDL designed the study, facilitated and participated in discussions around the study, drafted the first version of the manuscript, and wrote the final version of the manuscript. CLR merged the NOMAD and DPhacto cohorts, participated in discussions around the study, and critically revised the manuscript. SVT participated in discussions around the study and critically revised the manuscript. EC participated in discussions around the study and critically revised the manuscript. TL participated in discussions around the study and critically revised the manuscript. ML participated in discussions around the study and critically revised the manuscript. OSM participated in discussions around the study and critically revised the manuscript. MBJ acquired funding for the project, participated in discussions around the study, and critically revised the manuscript. NG participated in discussions around the study and critically revised the manuscript. CNR acquired funding for the project, participated in discussions around the study and critically revised the manuscript. AHO acquired funding for the project, designed the study, is Principal Investigator on the NOMAD and DPhacto cohorts, participated in discussions around the study, and critically revised the manuscript. All authors have read and approved the manuscript.

\section{Ethics approval and consent to participate}

The PODESA study has been tacitly approved by the Danish Data Protection Agency (file number 2013-10-11/104); this approval includes the use of register data. The DPhacto cohort was approved by the Danish Data Protection
Agency and the local Ethics Committee (file number H-2-2012-011) [37]. The NOMAD cohort was approved by the Ethics Committee for the Capital Region of Denmark (file number H-2-2011-047) [36]. All participants in the NOMAD and DPhacto studies received written and oral information about the projects, the practicalities of participating, potential risks of participating and having the possibility of withdrawal from the project without giving a specific reason with sufficient time for considerations of their participation. The persons agreeing to participate gave a written consent to participate in the study and the use of the data for research studies. PODESA is a merge of the similar NOMAD and Dphacto datasets for common analyses on the original research purposes of NOMAD and DPhacto, which has been tacitly approved by the Danish Data Protection Agency without the need of further specific consent by the participants (file number 2013-10-11/104).

\section{Consent for publication \\ Not applicable.}

\section{Competing interests}

The authors declare that they have no competing interests.

\section{Publisher's Note}

Springer Nature remains neutral with regard to jurisdictional claims in published maps and institutional affiliations.

\section{Author details}

${ }^{1}$ National Research Centre for the Working Environment, Lersø Parkallé 105, Copenhagen, Denmark. ${ }^{2}$ Section of Social Medicine, University of Copenhagen, Øster Farimagsgade 5, 1014, Copenhagen, Denmark. ${ }^{3}$ Department of Public Health and Primary Care, Ghent University, 4K3 (ingang 42), Corneel Heymanslaan 10, B-9000 Ghent, Belgium. ${ }^{4}$ Center for Social Medicine, Frederiksberg and Bispebjerg Hospital, Nordre Fasanvej 57, 2000 Frederiksberg, Denmark. ${ }^{5}$ Department of Public Health, Aarhus University, Bartholins Allé 2, 8000 Aarhus, Denmark. ${ }^{6}$ Department of Occupational and Social Medicine, Holbæk Hospital, Smedelundsgade 60, 4300 Holbæk, Denmark. 7Department of Forensic Science, University of Copenhagen, 2100 Copenhagen, Denmark. ${ }^{8}$ Department of Sports Science and Clinical Biomechanics, University of Southern Denmark, Campusvej 55, 5230 Odense, Denmark.

Received: 12 November 2018 Accepted: 22 February 2019 Published online: 04 March 2019

\section{References}

1. Nyhus M, Søgaard J. Rekonvalescens, sygemelding og sundhedsøkonomi [recovery, sick leave, and health economics]. Ugeskr Laeger. 2009;171(40): 2888-92.

2. The Danish Employment Ministry a. Analyse af Sygefraværet April 2008 [Analysis of Sickness Absence April 2008]. In: Ministry TDE. Copenhagen: The Danish Employment Ministry. p. 2008.

3. Lund T, Kivimäki M, Labriola M, Villadsen E, Christensen KB. Using administrative sickness absence data as a marker of future disability pension: the prospective DREAM study of Danish private sector employees. Occupational and Environmental Medicine. 2008;65(1):28-31. https://doi.org/10.1136/oem.2006.031393.

4. Lund T, Labriola M. Sickness absence in Denmark- Research, results, and reflections. Scand J Work Environ Health Suppl Scandinavian Journal of Work, Environment and Health, Supplement. 2009;7:5-14.

5. Kivimäki M, Forma P, Wikström J, Halmeenmäki T, Pentti J, Elovainio $M$, et al. Sickness absence as a risk marker of future disability pension: the 10-town study. J Epidemiol Community Health Journal of Epidemiology and Community Health. 2004;58(8):710. https://doi.org/10.1136/jech.2003.015842.

6. Bang Christensen $K$, Lund T, Labriola M, Villadsen E, Bültmann U. The fraction of long-term sickness absence attributable to work environmental factors: prospective results from the Danish Work Environment Cohort Study. Occupational and environmental medicine. 2007;64(7):487-9. https://doi.org/10.1136/oem.2006.028563.

7. Sterud T. Work-related mechanical risk factors for long-term sick leave: a prospective study of the general working population in Norway. Eur J Pub Health. 2014;24(1):111-6.

8. Andersen LL, Fallentin N, Thorsen SV, Holtermann A. Physical workload and risk of long-term sickness absence in the general working population and 
among blue-collar workers: prospective cohort study with register followup. Occupational and Environmental Medicine. 2016;73(4):246-53. https://doi.org/10.1136/oemed-2015-103314.

9. Alipour A, Ghaffari M, Shariati B, Jensen I, Vingard E. Four-year incidence of sick leave because of neck and shoulder pain and its association with work and lifestyle. Spine. 2009;34(4):413-8. https://doi.org/10.1097/BRS.0b013e3181948185.

10. Bot SD, Terwee CB, van der Windt DA, van der Beek AJ, Bouter LM, Dekker J. Work-related physical and psychosocial risk factors for sick leave in patients with neck or upper extremity complaints. International archives of occupational and environmental health. 2007;80(8):733-41. https://doi.org/10.1007/s00420-007-0186-z.

11. Labriola M, Lund T, Burr H. Prospective study of physical and psychosocial risk factors for sickness absence. Occupational Medicine. 2006;56(7):469-74 https://doi.org/10.1093/occmed/kql058.

12. Lund T, Labriola M, Christensen KB, Bültmann U, Villadsen E. Physical work environment risk factors for long term sickness absence: prospective findings among a cohort of 5357 employees in Denmark. BMJ. 2006. https://doi.org/10.1136/bmj.38731.622975.3A.

13. Elders LA, Heinrich J, Burdorf A. Risk factors for sickness absence because of low back pain among scaffolders: a 3-year follow-up study. Spine. 2003; 28(12):1340-6. https://doi.org/10.1097/01.BRS.0000065481.43111.7B.

14. Prince SA, Adamo KB, Hamel ME, Hardt J, Gorber S, Tremblay M. A comparison of direct versus self-report measures for assessing physical activity in adults: a systematic review. International Journal of Behavioral Nutrition and Physical Activity. 2008:5:56. https://doi.org/10.1186/1479-5868-5-56.

15. Sabia S, van Hees VT, Shipley MJ, Trenell MI, Hagger-Johnson G, Elbaz A, et al. Association between questionnaire- and accelerometer-assessed physical activity: the role of sociodemographic factors. Am J Epidemiol. 2014;179(6): 781-90. https://doi.org/10.1093/aje/kwt330.

16. Gupta N, Christiansen CS, Hanisch C, Bay H, Burr H, Holtermann A. Is questionnaire-based sitting time inaccurate and can it be improved? A cross-sectional investigation using accelerometer-based sitting time. BMJ Open. 2017;7(1):e013251. https://doi.org/10.1136/bmjopen-2016-013251 PubMed PMID: 28093433; PubMed Central PMCID: PMCPMC5253534.

17. Troiano RP, Berrigan D, Dodd KW, MÂSse LC, Tilert T, McDowell M. Physical activity in the United States measured by accelerometer. Med Sci Sports Exerc. 2008:40(1):181-8.

18. Gupta N, Heiden M, Mathiassen SE, Holtermann A. Prediction of objectively measured physical activity and sedentariness among blue-collar workers using survey questionnaires. Scand J Work Environ Health. 2016;42(3):237-45. https:// doi.org/10.5271/sjweh.3561 Epub 2016/04/14. PubMed PMID: 27067991.

19. Wiktorin C, Karlqvist L, Winkel J. Validity of self-reported exposures to work postures and manual materials handling. Stockholm MUSIC I Study Group. Scand J Work Environ Health 1993;19(3):208-214. Epub 1993/06/01. PubMed PMID: 8367699.

20. Gupta N, Heiden M, Mathiassen SE, Holtermann A. Is self-reported time spent sedentary and in physical activity differentially biased by age, gender, body mass index, and low-back pain? Scand J Work Environ Health. 2017. https://doi. org/10.5271/sjweh.3693 Epub 2017/12/01. PubMed PMID: 29184965.

21. Strath SJ, Kaminsky LA, Ainsworth BE, Ekelund U, Freedson PS, Gary RA, et al. Guide to the assessment of physical activity: clinical and research applications: a scientific statement from the American Heart Association. Circulation. 2013;128(20):2259.

22. Pedišić Z. Measurement issues and poor adjustments for physical activity and sleep undermine sedentary behaviour research - the focus should shift to the balance between sleep, sedentary behaviour, standing and activity. Kinesiology. 2014;46(1):135-46.

23. Pedisic Z, Dumuid D, Olds TS. Integrating sleep, sedentary behaviour, and physical activity research in the emerging field of time-use epidemiology: definitions, concepts, statistical methods, theoretical framework, and future directions. Kinesiology. 2017:49(2):252-69.

24. Gupta N, Mathiassen SE, Mateu-Figueras G, Heiden M, Hallman DM, Jørgensen MB, et al. A comparison of standard and compositional data analysis in studies addressing group differences in sedentary behavior and physical activity. International Journal of Behavioral Nutrition and Physical Activity. 2018;15(1):1-12. https://doi.org/10.1186/s12966-018-0685-1.

25. Aitchson J. The statistical analysis of compositional data; 1986.

26. Rasmussen CL, Palarea-Albaladejo J, Bauman A, Gupta N, Nabe-Nielsen K, Jørgensen MB, et al. Does Physically Demanding Work Hinder a Physically
Active Lifestyle in Low Socioeconomic Workers? A Compositional Data Analysis Based on Accelerometer Data. IJERPH International Journal of Environmental Research and Public Health. 2018;15(7):1306. https://doi.org/10.3390/ijerph15071306.

27. Hensing G. Chapter 4. Methodological aspects in sickness-absence research. Scandinavian Journal of Public Health. 2004;32(5):44-8.

28. Andersen LL, Clausen T, Persson R, Holtermann A. Dose-response relation between perceived physical exertion during healthcare work and risk of long-term sickness absence. Scand J Work Environ Health. 2012;38(6):582-9.

29. Labriola M, Christensen KB, Lund T, Nielsen ML, Diderichsen F. Multilevel analysis of workplace and individual risk factors for long-term sickness absence. J Occup Environ Med. 2006;48(9):923-9.

30. Sundstrup E, Hansen AM, Mortensen EL, Poulsen OM, Clausen T, Rugulies R, et al. Cumulative occupational mechanical exposures during working life and risk of sickness absence and disability pension: prospective cohort study. Scand J Work Environ Health. 2017;43(5):415-25. https://doi.org/10.5271/sjweh.3663 PubMed PMID: WOS:000408753300004.

31. Blank N, Diderichsen F. Short-term and long-term sick-leave in Sweden: relationships with social circumstances, working conditions and gender. Scand J Soc Med. 1995;23(4):265-72. https://doi.org/10.1177/ 140349489502300408 PubMed PMID: 8919369.

32. Taimela S, Justén S, Aronen P, Sintonen H, Läärä E, Malmivaara A, et al. An occupational health intervention programme for workers at high risk for sickness absence. Cost effectiveness analysis based on a randomised controlled trial. Occup Environ Med. 2008;65(4):242-8. https://doi.org/10.1136/oem.2007.033167.

33. Sundquist J, Al-Windi A, Johansson S-E, Sundquist K. Sickness absence poses a threat to the Swedish welfare state: a cross-sectional study of sickness absence and self-reported illness. BMC Public Health. 2007;7(1):45. https://doi.org/10.1186/1471-2458-7-45.

34. Krane L, Johnsen R, Fleten N, Nielsen CV, Stapelfeldt CM, Jensen C, et al. Sickness absence patterns and trends in the health care sector: 5-year monitoring of female municipal employees in the health and care sectors in Norway and Denmark. Hum Resour Health. 2014;12(1):37. https://doi.org/10.1186/1478-4491-12-37.

35. Tolonen A, Rahkonen $\mathrm{O}$, Lahti J. Leisure-time physical activity and direct cost of short-term sickness absence among Finnish municipal employees. Archives of Environmental \& Occupational Health. 2017;72(2):93-8. https://doi.org/10.1080/19338244.2016.1160863.

36. Gupta N, Christiansen CS, Hallman DM, Korshøj M, Carneiro IG, Holtermann A. Is objectively measured sitting time associated with low Back pain? A cross-sectional investigation in the NOMAD study. PLoS One. 2015;10(3): e0121159. https://doi.org/10.1371/journal.pone.0121159.

37. Jørgensen MB, Korshøj M, Lagersted-Olsen J, Villumsen M, Mortensen OS, Skotte J, et al. Physical activities at work and risk of musculoskeletal pain and its consequences: protocol for a study with objective field measures among blue-collar workers. BMC Musculoskelet Disord. 2013;14(1):213. https://doi.org/10.1186/1471-2474-14-213.

38. Gupta N, Jensen BS, Carneiro IG, Christiansen CS, Holtermann A, Jensen BS, et al. Face validity of the single work ability item: comparison with objectively measured heart rate reserve over several days. Int J Environ Res Public Health International Journal of Environmental Research and Public Health. 2014;11(5):5333-48.

39. Jorgensen MB, Gupta N, Korshoj M, Lagersted-Olsen J, Villumsen M, Mortensen OS, et al. Cohort profile: The Danish Physical activity cohort with Objective measurements (DPhacto). [Journal article]. In press.

40. Actilife software version 5.5. Florida, United States of America: Actigraph Corporation.

41. Skotte J, Korshøj M, Kristiansen J, Hanisch C, Holtermann A. Detection of physical activity types using triaxial accelerometers. J Phys Act Health. 2014; 11(1):76-84.

42. Ingebrigtsen JS, Ingunn; Christiansen, Caroline; Skotte, Jørgen; Hanisch, Christiana; Krustrup, Peter; Holtermann, Andreas. Validation of a Commercial and Custom Made Accelerometer-Based Software for Step Count and Frequency during Walking and Running. Journal of Ergonomics. 2013;3(2). doi: https://doi.org/10.4172/2165-7556.1000119.

43. Stemland I, Ingebrigtsen J, Christiansen CS, Jensen BR, Hanisch C, Skotte J, et al. Validity of the Acti4 method for detection of physical activity types in freeliving settings: comparison with video analysis. Ergonomics. 2015;58(6):953-65.

44. Gupta N, Dumuid D, Korshøj M, Jørgensen MB, Søgaard K, Holtermann A. Is Daily Composition of Movement Behaviors Related to Blood Pressure in 
Working Adults? Medicine \& Science in Sports \& Exercise. 2018;50(10):21505. https://doi.org/10.1249/mss.0000000000001680 PubMed PMID: 00005768201810000-00020.

45. Denmark S. FRAN - Fravær ansættelsesforhold [FRAN - Absence employment type] Denmark: Statistics Denmark; 2017 [cited 2017 29-092017]. Available from: https://www.dst.dk/extranet/ForskningVariabellister/ FRAN\%20-\%20Fravær\%20ansættelsesforhold.html. Cited 26 Feb 2019.

46. Denmark S. FRPE - Fravær fraværsperioder [FRPE - Absence Periods of absence] Denmark: Statistics Denmark; 2017 [cited 2017 29-09-2017]. Available from: https:/www.dst.dk/extranet/ForskningVariabellister/ FRPE\%20-\%20Fraværsperioder.html. Cited 26 Feb 2019.

47. Statistics Denmark a. Data fra andre styrelser [data from other agencies] [cited 2018 23-10-2018]. Available from: https://www.dst.dk/da/TilSalg/ Forskningsservice/Data/Andre_Styrelser.

48. Hjollund NH, Larsen FB, Andersen JH. Register-based follow-up of social benefits and other transfer payments: accuracy and degree of completeness in a Danish interdepartmental administrative database compared with a population-based survey. Scandinavian Journal of Public Health. 2007;35(5): 497-502.

49. The Danish Agency for Labour Market and Recruitment a. DREAM vejledning version $42 \mathrm{~V} 2$ [DREAM guide version 42 version 2]. Recruitment TDAfLMa; 2018 22-08-2018. Report No.

50. Statistics Denmark a. Statistical presentation [of the absence statistics]: Statistics Denmark; [cited 2018 06-11-2018]. Available from: https://dst.dk/ en/Statistik/dokumentation/documentationofstatistics/absence/statisticalpresentation.

51. Statistics Denmark a. Absence [cited 20187 September]. Available from: https://dst.dk/en/Statistik/dokumentation/ documentationofstatistics/absence.

52. Thorsen SV, Pedersen J, Kristiansen J, Flyvholm M-A, Bültmann U. Perceived work-related stress and sickness absence: A prospective study of 10047 female and 6897 male Danish employees. [Journal article draft]. In press.

53. Thorsen SV, Flyvholm M-A, Bültmann U. Self-reported or register-based? A comparison of sickness absence data among 8110 public and private employees in Denmark. Scand J Work Environ Health Scandinavian Journal of Work, Environment \& Health. 2018;44(6). http://www.sjweh.fi/show_ abstract.php?abstract id $=3747$.

54. Dumuid D, Stanford TE, Martin-Fernandez JA, Pedisic Z, Maher CA, Lewis LK, et al. Compositional data analysis for physical activity, sedentary time and sleep research. Stat Methods Med Res 2017:962280217710835. Epub 2017/ 05/31. doi: https://doi.org/10.1177/0962280217710835. PubMed PMID: 28555522 .

Ready to submit your research? Choose BMC and benefit from:

- fast, convenient online submission

- thorough peer review by experienced researchers in your field

- rapid publication on acceptance

- support for research data, including large and complex data types

- gold Open Access which fosters wider collaboration and increased citations

- maximum visibility for your research: over $100 \mathrm{M}$ website views per year

At $\mathrm{BMC}$, research is always in progress.

Learn more biomedcentral.com/submissions 